\title{
Design Examination through Pattern Characteristic Identification: A New Kain Punca Potong (KPP) Weaving Design
}

\author{
Nani Hartina Ahmad, Nazlina Shaari, Noor Azizi Mohd Ali, Muhammad Pauzi Abd Latif
}

\begin{abstract}
The weaving industry is regarded as one of the world's most popular industries. Clearly, designers' ideas gave them a drive to start a conversation about the future of design studies and research training, as well as practicing a fashion that illuminated this industry. Vigorous efforts are being exerted into producing new processes, with intensifying designing endeavors from design motifs of textile design. This paper presents a detailed study on weaving design which functions as a new approach to KPP weaving design process by defining pattern characteristics of woven fabric design. Generally speaking, there is no evidence that KPP design identity characteristics can be implemented solely on the basis of the idea process. A literature review was performed to define the current framework relevant to the choice of design and motifs and the implementation of pattern recognition characteristics for KPP in the weaving industry. It is shown that weaving design patterns and characteristics can be used as a single review point and should therefore be used as KPP's review technique for this research. When attempting to identify design innovation and motif selections in fabric weaving, a design framework for design identification through pattern characteristics is significant. The result showed that the design framework generated from this study will provide the overall aesthetic value for KPP pattern characteristics, which can serve as a new approach in weaving design and design studies, with the potential for further development suitable for future research, as suggested from the results of this study.
\end{abstract}

Keywords: Design framework, design studies, pattern characteristic, textile design, weaving design

\section{INTRODUCTION}

Malaysia is a country deeply steeped in culture, with exquisite woven textile representing one of them. The nation is well known for its rich cultural heritage, where the Malay heritage's esthetic principles are beautifully expressed in most of its brand designs for handicrafts. Weaved textiles are legendary living arts that have played a major role in enriching the cultural development of Malaysia. The beauty of textile weaving is one of Malaysia's most sought-after handicrafts, due to its beautiful designs, patterns and motifs that have been passed down to their descendants from traditional weavers and subsequently embraced and changed

Revised Manuscript Received on September 14, 2019.

Nani Hartina Ahmad, Faculty of Design and Architecture, Universit Putra Malaysia (UPM), Serdang, Selangor, Malaysia \& Faculty of Art and Design, Universiti Teknologi MARA (UiTM) Kelantan, Machang, Kelantan, Malaysia. Email: fatina8277@ gmail.com

Nazlina Shaari, Faculty of Design and Architecture, Universiti Putra Malaysia (UPM), Serdang, Selangor, Malaysia. Email: nazlinashaari@upm.edu.my

Noor Azizi Mohd Ali, Faculty of Design and Architecture, Universiti Putra Malaysia (UPM), Serdang, Selangor, Malaysia. Email nooraz@upm.edu.my

Muhammad Pauzi Abd Latif, International Institute of Islamic mdpauzi@yahoo.com.my Civilisation and Malay World (ISTAC), Kuala Lumpur, Malaysia Email

by modern designers. The fabrics reflect society's response, whilst showing the improvements in dyeing, raw materials, and process techniques that have occurred. The surroundings, the weaver, and various aspects of the daily life of the weaver largely influenced the motifs and patterns used in textile items. The fabrics had a profound meaning in the weavers and wearers' social and cultural lives [1].

The elegance of Kain Punca Potong (KPP)'s own distinctive style comes from the combination of ikat limar technique, which is visible on the fabric body or the end of the cloth. Historically in Kelantan, the ruler Cik Siti Wan Kembang (1610-1677) also wore KPP, which dates back to her 17 th century rule. KPP is a long fabric traditionally crafted using the ikat technique in square patterns with striped motifs consisting of a decorative limar pattern. Generally, this fabric is woven with bright threads [2] Traditionally, in the yarn phase, weaving pattern recognition of woven fabrics is typically very subjective to analyze. The step-in weaving is to ensure that enough yarns are measured before weaving starts. To ensure that the process is right and runs smoothly, it is important to measure the length of the warp and weft required. Several researchers have therefore focused on finding new knowledge that can be used to automatically identify weave patterns to improve the efficiency of textile production [3].

The weaving process followed two primary routes, known as the design process and the technical process. Treating this as a starting point, is important for the study to recognize the pattern and characteristics of KPP while trying to produce woven fabric, knowledge of traditional weaves, style, and weaving techniques. A study of weaving design patterns and characteristics are general aspects of design needs and are an essential requirement that must be met to define the weaving design process successfully. The term "pattern" means simply repeating more than one design element working together. The patterning system can be represented as serial processing or as a parallel process within and between the processes of design. This study resulted in several types of patterns being formed in checkered design, with the introduction of design framework identification. That said, this work has provided a set of criteria for reporting strategy purposes that is coupled with observation-including study design that is appropriate for use when attempting to identify KPP weaving model characteristics.

Weaving patterns are typically designed to achieve aesthetics or practical characteristics. Therefore, understanding of weaving patterns is one of the most 


\section{Design Examination through Pattern Characteristic Identification: A New Kain Punca Potong (KPP) Weaving Design}

important factors in the process of textile design. Weave design is one of the most important steps in the processes of textile production. It makes it possible to build a textile structure that fits the original design concept [4].

This study chose to investigate old woven textiles lost in the sands of time, where investigating Kelantan KPP's weaving art proves to be difficult as its restricted sources of information demanded an in-depth investigation from different angles and perspectives. Compared to a few other woven cloths from other nations, our current culture has never been properly exposed and familiarized with the pattern characteristics of Kelantan's design identity. Since the interest in inheriting weaving skills among younger generations is waning, these patterns and motifs must be preserved so that they can be enjoyed by the public, especially by future generations.

\section{RELATED WORK}

This section summarizes relevant studies on pattern compatibility, weaving design enhancement and approaches to weaving design.

\section{A. Pattern Compatibility}

In many cases, a pattern can be formed by techniques such as formation patterns and pattern typology. The pattern in Malay is called 'corak'. There are 3 forms of KPP's structures and patterns,' which can be defined as the body of the cloth in the middle of the fabric, the side border of the cloth, and the last part of the fabric called the end of the cloth. The combinations of checkered, striped and ikat 'limar' (weft ikat) designs made up the KPP patterns. Any textile designer is required to possess an innovative idea on how their design is going to look like as a finished fabric. Essentially, KPP is built with a line and space sensitivity that is well balanced across the checkered or stripped pattern's rectangular shape. The KPP is especially unique because of its checkered patterns weaved with ikat limar [5]. Pattern features provide essential product and component design information that significantly influences the functions, reliability, and quality of the product. Identifying pattern characteristics for a textile fabric will help designers concentrate on design studies during process design, thereby rapidly developing design schemes based on their close relationship to fabric functions, efficiency and quality [6].

The woven pattern is an important feature of woven fabrics and it can be produced to play a crucial role in the appearance of fabrics and its physical properties in any way. In this context, however, patterns primarily apply to the structure that transforms the identification of fabric patterns into an important part of textile weaving. Essentially, pattern detection is accomplished by classifying the parameters for full pattern recognition. The recognition process would define the texture characteristics of the fabric extracted by controlling the pattern arrangement in the fabric structure. Stylistic pattern order in the fabric was done to accomplish the identification and classification of woven. In [7] suggested a pattern recognition classification approach that could be divided into a type that is known as "supervised learning" and "unsupervised learning" in pattern formation.
Thus, in KPP structural pattern construction is based on unsupervised learning in the making fabrics.

\section{B. Weaving Design Enhancement}

The term 'weaving design' refers to the utilization of elements of technical design that have been further divided into several categories. In [8] suggested a system for weave pattern indexing and searching. In this article, they began by classifying the weave pattern into three categories based on the weave pattern description and the weave point distribution features, using the weave pattern smoothness and connectivity as parameters. Recognition of textile weaving patterns has attracted many researchers since the mid-1980s. The field's problem is mainly about how they initiate the weaving processes by designing the detection that deals with interlacing areas between warp and weft yarn in the fabric making [9].

\section{Weaving Design Approach}

Design is defined as an innovative, scientific, and complex process based on several qualities of design thinking. The pattern of design should be described in an understandable way. It was also carried out to understand the problems in depth and to establish suitable solutions. To ensure that the design patterns produced are accurate and long-term relevant, they should be independent of specific design fabric implementations. This is because pattern designing is very precise when it comes to examining the pattern arrangement of shapes and lines [10]. KPP flaunt different structures compared to other fabrics. The pattern creation phase in KPP involves the design, pattern and stylistic pattern order typology. Pattern characters have also been thoroughly delved into in KPP's development. This review ends with the specifics of a specially established design framework for KPP analysis. The Malays place very high value for hand-woven cloth, referring to how the fabrics are made in keeping with the design pattern and motifs. They weave the fabric with their heart and soul, taking into consideration their surroundings and simple lifestyle and reverently use human body parts as name to parts of the woven fabric. The textile structure is divided into two main parts, the main body and the end fabric. KPP has both ends free with elaborated designs known as the 'punca kain'.

In this study, KPP represents a part of local Malay heritage linked to the highest aesthetics due to its decorative style and exquisiteness that showcases Malay community artistic arts and unique pattern that is undoubtedly infused with heritage, since KPP is a traditional Malay art that is passed down from generations. One might argue that the Malay heritage of art is vast and covers the whole aspect of life through the slip of time, the experience of occupation and the changes in modern life that spread it far and wide. The other interesting part of the design of the fabric can be seen by patterns that are well arranged by the weavers to include the motifs and patterns that could be described in translations, rotations, reflections and glide reflections [11]. 


\section{METHODOLOGY}

A literature review was performed, in which it critically explores the characteristics of the pattern and weave design studies that is related to the identification of design characteristics from another region. A collection of revised mapping guidelines has been provided for review in this review process. This study includes the curating of all previous studies that are related to researches that have been identified from initial reviews namely online journals that focuses on pattern characteristics in weaving design. The search covered the titles, abstracts and keywords as stated according to the $\mathrm{K}-\mathrm{J}$ method.

\section{Previous Studies}

The following databases were searched electronically through Google Scholar, Science Direct, Emerald Insight, IEEE, Scopus and others. To ensure full coverage of the electronic database during literature search, specific keywords were used. This study includes the search for all previous studies discovered via reviews, namely online journals that concentrate on pattern characteristics of weaving design.

\section{E. Professional Discussion}

For this research, potential participants (field of expertise: relevant academicians, culture experts, collectors, curators, and designers) were contacted through e-mail and via the telephone and consequently extended an invitation to partake in the study. Upon receipt of a participant's consent to take part in the study, each participant was asked to provide written responses (via e-mail) to questions pertaining the participant's educational background, years of experience in textile design, and current employment

\section{F. Form Plan}

A design framework was developed in order to facilitate a design that can be described as a type of pattern, which was briefly introduced, on top of certain matters relating to the review of this identification method that were being discussed including style, aesthetic value, function and personality criteria in weaving design. Hence, a design framework was utilized in order to identify the method of identity identification. However, in order to identify identity, it is necessary to first identify the characters of work or handcraft, which in this case are applicable to KPP motifs, colors, style and function character identification.

\section{RESULTS AND DISCUSSION}

The curating process of relevant literatures was performed through the use of selected keywords search. The inclusion criteria for selected keywords considered during the literature review were words closely related to the patterns and characteristics of weaving design, especially those based on checkered design. In general, methodologies for searching and data extraction based on keywords are quite alike, but some keyword approaches brought phrases with different meaning that was quite unrelated to our review process. When we intend to identify the scope of a topic and classify the studies that exists in that particular scope, it is a given for us to use the selected precise keyword position.

relevant for our studies. On the other hand, some of it can provide good general information on one of the topics in our studies.

This study highlights the importance of the developed design study framework implementation in the research. To achieve this, this study reviewed pattern characteristics of weaving, where the general aspects of design critically characterized all weaving tasks and is an important condition for identifying the process of weaving design. We have shown that design and characteristics use is quite widespread, although its utilization is often unconsciously carried out and unsystematic. This study also has successfully developed a design framework based on the identification of patterns, design, and characteristics of KPP. There is a 3 step framework that should be followed when intending to review the design of KPP, which are: the establishment of pattern types, identifying the design and lastly, the study of KPP pattern characteristics selection criteria like style, aesthetic value, function and finally, the personality of the fabric.

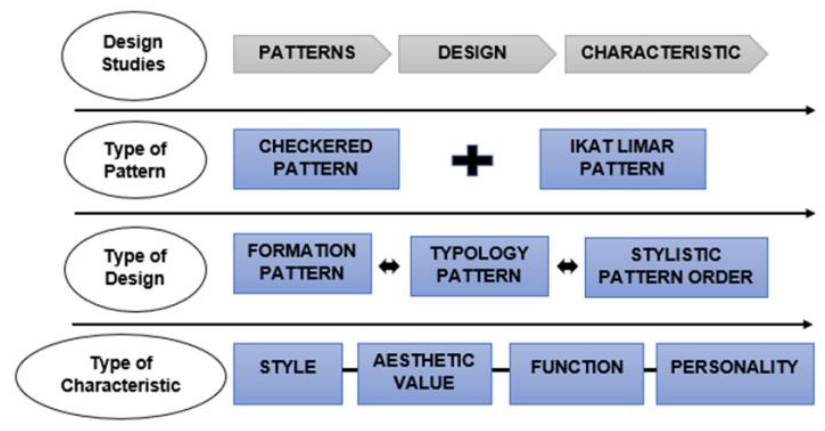

Fig. 1. Framework for KPP design studies identification

The research is resulted to help designers, researchers, educators and practitioners define design features based on the design framework in addition to promoting KPP adoption in the future. KPP is a traditional Malay woven textile that comes into being through vibrant color combinations. This cloth makes up the attire of men and women of Kelantan. The exquisiteness and beauty of KPP can be appreciated due to its combination with ikat limar technique that manages to enhance its beauty further. Design goals can be defined as the development goals and decisions taken in each design phase. They direct the choice of steps to be taken at each stage during the fabric design process [12].

The complexity of the design process will be explored to better understand how these factors affect the ability of a designer to function efficiently during designing. It allows the joint creation of a project scenario and thus sketches the solution between users and designers by engaging in this process through negotiation and mutual understanding of local craft identity and esthetic values [13]. The design process has become a profession, rooted primarily in the practices of the arts and crafts and - later in its growth - an 


\section{Design Examination through Pattern Characteristic Identification: A New Kain Punca Potong (KPP) Weaving Design}

academic discipline. In more recent times, designing work has been discovered to function as a central driver for social and economic innovation, which now in turn must clarify its position in the university context [14]. Designs can fulfill functional requirements but are difficult to manage or is quite unfeasible to handle. Designers depend on the system to enhance the manufacturing performance of the designs, so it is paramount importance that they update their designs and provide input relevant to manufacturing [15].

\section{CONCLUSION}

In this research, emphasis was placed on patterns and characteristics of weaving design review, where general design features that inevitably characterize all weaving tasks are an essential condition for weaving design process identification. We have shown that use of patterns and identity features is quite common, although often unconscious and unsystematic. Through a design framework based on the identifying process of KPP weaving patterns and characteristics reflect the importance of proper pattern construction in developing the fabric identity. The overall result showed that specific checkered design studies can be more in-depth in the effort to improve future research so that existing specific knowledge studies can be improved and thus help to expand the awareness of new designers on how to handle and build KPP designs in new styles but still in line with the original concept. It seeks to better the understanding of various design combinations aspects considered necessary for the ongoing process of actual designing.

\section{REFERENCES}

1 N. Jamil, T. M. Tengku Sembok, and Z. Abu Bakar. "Digital archiving of traditional songket motifs using image processing tool." Recent Researches in Chemistry, Biology, Environment and Culture (2011): 33-39.

2 Siti Zainon Ismail. Pakaian cara Melayu, Universiti Kebangsaan Malaysia. 2009: pp. 146.

3 Zhang, Jie, Binjie Xin, and Xiangji Wu. "A review of fabric identification based on image analysis technology." Textiles and Light Industrial Science and Technology 2, no. 3 (2013): 120-130.

4 Ng. Frankie MC, and Jiu Zhou. "Digital Jacquard textile design in a colorless mode." Research Journal of Textile and Apparel 10, no.2 (2006): 36-42.

5 Norwani Md Nawawi. Ikat limar the ancient Malay textile. Dewan Bahasa dan Pustaka. Kuala Lumpur, 2016: pp. 107-111.

6 Han, Xin, Rong Li, Jian Wang, Shengfeng Qin, and Guofu Ding. "Identification of key design characteristic for complex product adaptive design." International Journal of Advanced Manufacturing Technology 95, no. 1-4 (2018): 1215-1231.

7 Zhang, Rui, and Binjie Xin. "A review of woven fabric pattern recognition based on image processing technology." Research Journal of Textile and Apparel 20, no. 1 (2016): 37-47.

8 Zheng, Dejun, George Baciu, and Jinlian Hu. "Accurate indexing and classification for fabric weave patterns using entropy-based approach." 8th IEE International Conference on Cognitive Informatics, pp. 357-364, 2009.

9 Shidanshidi, Hooman, Fazel Naghdy, Golshah Naghdy, and Diana Wood Conroy. "3D geometric and haptic modeling of hand-woven textile artifacts." Entertainment Computing Symposium, pp. 87-100, 2010.

10 Schwinn, Alexander, and Joachim Schelp. "Design pattern for data integration." Journal of Enterprise Information Management 18, no. 4 (2005): 471-482.

11 Nawawi, Norwani Md, Rafeah Legino, Norakmal Abdullah, Mohd Firdaus Md Khalid, Ruzaika Omar Basaree, and Mohd Yusof Ahmad. "Symmetrical pattern: Analysing Songket in wallpaper patterns." International Colloqium of Art and Design Education Research, pp. 323-336, 2015.

12 Evbuomwan, N.F.O, Sangarappillai Sivaloganathan, and A. Jebb. "A survey of design, philosophies, models and systems." Proceeding of the Institution of Mechanical Engineering, Part B: Journal of Engineering Manufacture 210, no.4 (1996): 301-320.

13 Ebenreuter, Natalie. "The dynamic of design." Kybernets 36, no. 9/10 (2007): 1318-1328

14 Glanville, Ranulph, and Wolfgang Jonas. "Research through design through research," Kybernetes (2007).

15 Barnawal, Prashant, Micheal C. Dorneich, Frank Peters, and Matthew C. Frank. "Design and Evaluation of Designer Feedback System in Design for Manufacturability." Human Factors and Ergonomics Society Annual Meeting, vol. 59, no. 1, pp.1142-1146, 2015.

\section{AUTHORS PROFILE}

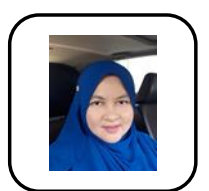

Nani Hartina Ahmad

BA (Hons) in Textile Design and MA in Design Technology, PhD student in the Integrated Design Studies at Universiti Putra Malaysia. Field of Expertise: Textile Design / Weaving Design.

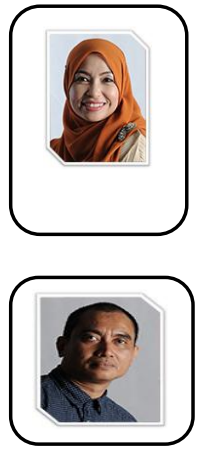

Assoc. Prof. Dr. Nazlina Shaari

$\mathrm{PhD}$ in Kansei (Artifact Design), Chiba University Japan. Field of Expertise: Kansei in Artifact Design / Textile \& Fashion Design / Design Emotion / Materials Planning.

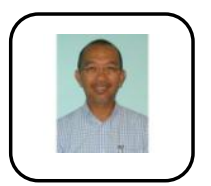

Assoc. Prof. Dr. Muhammad Pauzi Abd Latif $\mathrm{PhD}$ in Human Communication, Universiti Putra Malaysia. Field of Expertise: Economic, Business and Management / Marketing / Communications. 